\title{
Surface initiated polymerization from integrated poly(dimethylsiloxane) enables crack-free large area wrinkle formation
}

\author{
Zhong Li ${ }^{a, b, c}$, Shen Zhang ${ }^{a}$, Pengfei Zhang ${ }^{a}$, Dayong Yang ${ }^{a}$, Gang Jin ${ }^{c, d}$ \\ and Hongwei $\mathrm{Ma}^{\mathrm{a} *}$
}

\begin{abstract}
We conducted surface initiated polymerization from stretched poly(dimethylsiloxane) to construct a polymer/PDMS bilayer structure, which formed crack-free wrinkles over large area $\left(>6 \mathrm{~cm}^{2}\right)$ upon recovery from the stretched state. This system further allowed us to reveal the dynamics and memory effect of wrinkling. Copyright $\odot 2011$ John Wiley \& Sons, Ltd.
\end{abstract}

Supporting information may be found in the online version of this article.

Keywords: wrinkle; poly(dimethylsiloxane); surface initiated polymerization; dynamics; crack-free

\section{INTRODUCTION}

Studies based on natural ${ }^{[1-4]}$ and artificial systems ${ }^{[5-11]}$ have partially revealed the underlying mechanisms of the wrinkling phenomenon. Recently, these research efforts were paid off as evident from demonstrated applications based on wrinkles, such as tools for analyzing mechanical properties of films at the nanometer scale, ${ }^{[12-14]}$ fabricating flexible microelectronics, ${ }^{[15,16]}$ microlens arrays, ${ }^{[17]}$ and anti-reflective substrates in photonics. ${ }^{[18]}$ Despite significant progress made for the past $~ 10$ years, we identified the following challenges as obstacles to the success of future wrinkle applications: (i) how to make wrinkles on large surface area, which depended on technologies of bilayer construction and force exerting. Most existing methods produce parallel wrinkles with area below $1 \mathrm{~cm}^{2}$; (ii) how to reduce/eliminate defects that seemed to be inevitable during the process of wrinkle formation. Cracks and forks are the two typical defects found in resulted wrinkling area. ${ }^{[6,8,12,19-22]}$ Common causes for the formation of cracks are that the upper-skins are too fragile to sustain both large dimensional change and small lateral outward extension.

Herein, we report a new system (Scheme 1) that utilizes surface initiated polymerization (SIP) to generate in situ a polymeric upper-skin on a stretched elastic foundation, which is initiator integrated poly(dimethylsiloxane) (iPDMS). ${ }^{\left[{ }^{[23,24]}\right.}$ Upon release, the stretched iPDMS elastomer returns to its original length, thus triggers the dimensional mismatch, subsequently lead to the wrinkle formation. This system allows the formation of ordered and tunable wrinkling patterns at the micro-scale and nano-scale over large areas $\left(>6 \mathrm{~cm}^{2}\right)$. It also allows us to monitor the dynamics of wrinkling, which is in favor of revealing the nature of defect formation and eventually eliminating those defects.

\section{EXPERIMENTAL}

Integrated poly(dimethylsiloxane) sheet was prepared by mixing a vinyl-terminated initiator (undec-10-enyl2-bromo-2- methylpropanoate, $C$ ) with the viscous base $(A)$ and curing agent (B) of Sylgard 184 at a desired ratio $(A / B / C=10: 1: 0.5)$, which was cured at $65^{\circ} \mathrm{C}$ for $3 \mathrm{~h}$. The resulted iPDMS sheet was then stretched (Fig. 1e) and subjected to SIP for polymer coating.

For SIP of 2-hydroxyethyl methacrylate (HEMA), a transparent, pale-blue solution was prepared first by adding $\mathrm{CuCl}_{2}(27.3 \mathrm{mg}$, $0.16 \mathrm{mmol}$ ), 2,2'-bipyridine (Bipy, $50 \mathrm{mg}, 0.32 \mathrm{mmol}$, i.e., $1: 2 \mathrm{~mole}$ ratio), and monomer HEMA $(2.08 \mathrm{~g}, 16 \mathrm{mmol})$ to $36 \mathrm{ml}$ of Milli-Q water and $4 \mathrm{ml}$ of methanol. After deoxygenating for $5 \mathrm{~min}$, the preceding blue solution and a colorless ascorbic acid $(28.2 \mathrm{mg}$, $0.16 \mathrm{mmol}$ ) were mixed for $25 \mathrm{~min}$. The final mixture was red in color due to the reduction of the deactivator $\mathrm{Cu}(\mathrm{III}) / \mathrm{Bipy}$ complex to activator $\mathrm{Cu}(\mathrm{l}) / \mathrm{Bipy}$ complex. The stretched iPDMS sheet was then immersed in the red mixture under an argon atmosphere for SIP.

For SIP of oligo(ethylene glycol) methacrylates (OEGMA), $20 \mathrm{ml}$ of methanol and $20 \mathrm{ml}$ of Milli-Q water were mixed and OEGMA $(8.42 \mathrm{~g}, 16 \mathrm{mmol})$ was added. Most reaction conditions were the same as that of HEMA except the SIP time was longer because methanol was known to deaccerlate SIP. For the cross-link

\footnotetext{
* Correspondence to: Prof. Hongwei Ma, Division of Nanobiomedicine, Suzhou Institute of Nano-tech and Nano-bionics, Chinese Academy of Sciences, Suzhou, 215125, China.

E-mail: hwma2008@sinano.ac.cn

a Z. Li, S. Zhang, P. Zhang, D. Yang, H. Ma

Division of Nanobiomedicine, Suzhou Institute of Nano-tech and Nano-bionics, Chinese Academy of Sciences, Suzhou, 215125, China

b Z. Li

Graduate University of the Chinese Academy of Sciences, Beijing, 100049, China

c Z. Li, G. Jin

Institute of Biophysics, Chinese Academy of Sciences, Beijing, 100101, China

d G. Jin

Institute of Mechanics, Chinese Academy of Sciences, Beijing, 100191, China
} 


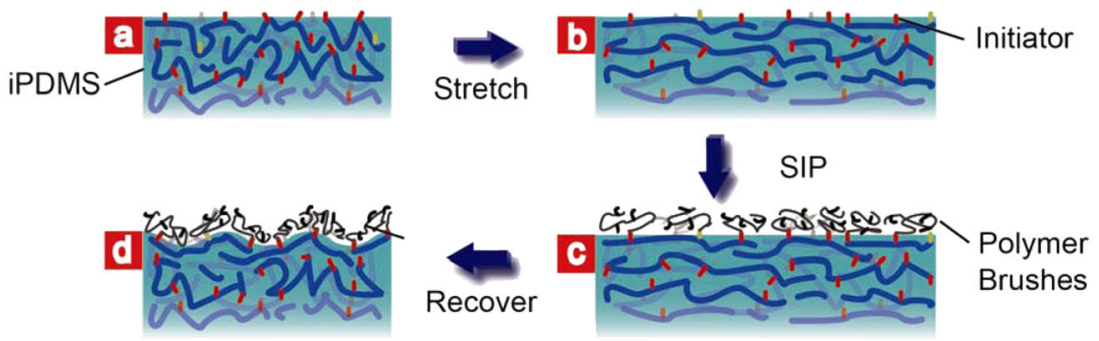

Scheme 1. Outline of the wrinkle fabrication process. (a) integrated poly(dimethylsiloxane) (iPDMS) was prepared via standard thermo curing and the initiators was covalently integrated into the 3-dimensional network of PDMS; (b) stretching was expected to bring some of the buried initiators (colored with yellow) up to the surface of iPDMS slab, making them available for surface initiated polymerization (SIP); (c) SIP led to a polymer layer coating on iPDMS; and (d) recovery of the foundation (iPDMS) triggered the wrinkling process.

experiments, the SIP solution was prepared as above except that cross-linker ethylene dimethacrylate $(0.317 \mathrm{~g}, 1.6 \mathrm{mmol})$ was added.

We used optical microscope (OLYMPUS, BX-51; Olympus Corporation, Tokyo, Japan), scanning electron microscopy (SEM; FEl, Quanta 400; FEG, Inspect S; Hillsboro, OR), and atomic force microscopy (AFM; Veeco, Dimension 3100; Santa Barbara, $C A)$ to observe the morphology of wrinkles.

\section{RESULTS AND DISCUSSION}

It was obvious from Fig. 1a that this strategy could fabricate large area of wrinkles. After SIP and prior recovering, the now polymer coated and stretched iPDMS slab was transparent. It gradually became frosted along the recovering process as previously reported, ${ }^{[5]}$ which was a direct consequence of wrinkle formation. Thus, one could easily identify the area of wrinkles by observing the frosted area. Given that the whole fabrication process (i.e., the duration of which iPDMS slab was stretched) was typically less than $24 \mathrm{~h}$, the aging/relaxation issue of rubber was negligible. We varied monomers, SIP time, $\varepsilon\left(\varepsilon I / I_{0}\right.$, where $I$ and $I_{0}$ are the original and stretched length of iPDMS slab) and initiator feed ratio of iPDMS ( $R_{\text {initiator }}$ ) to alter the characteristics of resulted wrinkles (e.g., wavelength $\lambda$ ), which was mainly characterized by AFM and SEM.

Figure $1 \mathrm{~b}$ was a SEM image of wrinkles prepared using the following conditions: monomer HEMA, 15 min of SIP, $R_{\text {initiator }}=$ 0.5 , and $\varepsilon=2.0$. We further applied AFM to obtain $\lambda$ (Fig. 1c and d). The wrinkle in this study was typically consisted of parallel ridges and trenches of micrometer scale, which was perpendicular to the direction of stretching. The value of $\lambda$ was defined as the distance between two adjacent peaks, which was $\sim 2 \mu \mathrm{m}$ with amplitude close to $600 \mathrm{~nm}$. We found that the profile of wrinkle shows convex crests with the sharp valleys rather than the smooth sinusoidal shape, which was attributed to the dull apex of the AFM-tip. However, such behavior may also indicate significant differences in the wrinkling behavior of this SIP/iPDMS system as compared with a conventional polymer film. Figure $1 \mathrm{f}-\mathrm{i}$ presented how monomer, SIP time, $\varepsilon$, and $\mathrm{R}_{\text {initiator }}$ alter the $\lambda$ of resulted wrinkles.

Although all other conditions were kept the same, the increase of $\mathrm{R}_{\text {initiator }}$ increased $\lambda$, especially when $\mathrm{R}_{\text {initiator }}$ was larger than 0.6 (Fig. 1f). Although all other conditions were kept the same, unlike the past researches, the increase of $\varepsilon$ decreased $\lambda$, especially for $\varepsilon>1.6$ (Fig. 1g). Although $\varepsilon$ and $\mathrm{R}_{\text {initiator }}$ were kept the same, the increase of SIP time increased $\lambda$ (Fig $1 \mathrm{~h}$ and i). Note that OEGMA experienced slower polymerization kinetics, so we applied longer SIP time to achieve visible wrinkles. To summarize, SIP time and $\mathrm{R}_{\text {initiator }}$ had positive correlation with $\lambda$ whereas $\lambda$ had negative correlation with $\lambda$. These trends could be explained by the following equation and model.

Previous studies concluded eqn. (1) to predict the wrinkling behavior: ${ }^{[1,2,4,5]}$

$$
\lambda=4.36 h\left(\frac{E_{S}\left(1-v_{p}^{2}\right)}{E_{P}\left(1-v_{S}^{2}\right)}\right)^{1 / 3}
$$

where the subscripts $s$ and $p$ refer to the top surface and the PDMS membrane, respectively; $h(\mathrm{~m})$ is the thickness of the top surface (i.e., the poly(HEMA) or poly(OEGMA) coatings), $E(\mathrm{~Pa})$ is the Young's modulus, and $v$ (unitless) is the Poisson's ratio. Equation (1) predicts that an increase of $h$ will increase $\lambda$, which was the case in Fig. $1 \mathrm{~h}$ and $\mathrm{i}$ because longer SIP time led to a thicker polymer coating. If one knew the value of $E_{s}$ and $E_{p}$, Eqn. (1) could also be used to determine the value of $h$ (Fig. S2). ${ }^{[13]}$

Although Eqn. (1) was useful, it could not be directly applied to explain how $\mathrm{R}_{\text {initiator }}$ and $\varepsilon$ affected the wrinkling in the case that the polymer-iPDMS system was very different from the AuPDMS system. ${ }^{[25]}$ Alternatively, we proposed the following mechanism that could explain Fig 1f-1g. From Fig. 1f, we knew that the increased initiator density will increase $\lambda$. From Fig. 1g, we observed $\lambda$ decreased as $\varepsilon$ increased, indicating the actual initiator density decreased as $\varepsilon$ increased. Although the number of initiator increased because the stretching of iPDMS will cause extra initiators to be exposed at the surface of iPDMS (Scheme 1a-b), the area increased much more that led to a net decrease of initiator density. Compare with the relative acutely variation of $\lambda$ by manipulating $R_{\text {initiator, }}$ a conclusion that stretching can only brought slight change in initiator density on the surface of iPDMS was deduced, which can offer us deeper insight into this material.

Although uniaxial stress is commonly used to trigger wrinkle formation, researchers rarely employed drastic deformation so to avoid crack formation. ${ }^{[6-8,19,20,22]}$ In Fig. 2a-c, we demonstrated that only a small stress could lead to severe crack formation if crosslinking agent was added during the SIP. Given the large value of $\varepsilon=2.0$, the iPDMS slab experienced a width narrowing (i.e., a lateral inward contraction) perpendicular to the stretching direction (Fig. 2a). Upon recovery, the now crosslinked polymer layer coated iPDMS slab underwent width recovery (i.e., lateral outward extension), which caused the polymer layer to be stretched and subsequent formation of cracks (Fig. 2b-c). If such lateral outward extension was removed, one observed minimal crack formation even for crosslinked upper-skin (Fig. 2d-e). Considering the different interaction between grafted polymers with crosslinker, a model (Fig. S3), uncrosslinked polymer skin 

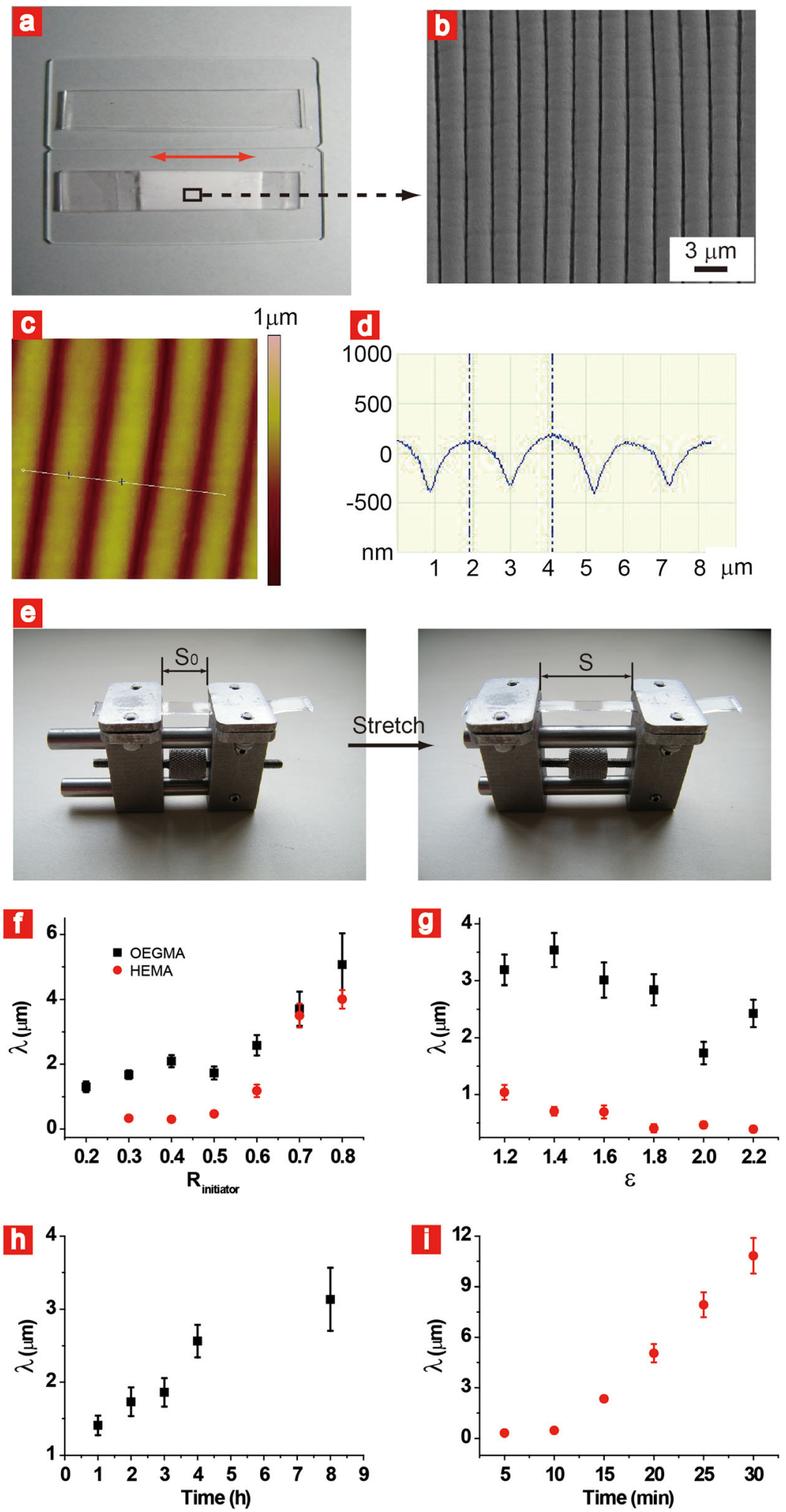

Figure 1. Morphological characterization of a poly 2-hydroxyethyl methacrylate (HEMA)/integrated poly(dimethylsiloxane) (iPDMS) bilayer with wrinkles. (a) Digital camera images of iPDMS slab before (upper) and after (lower) wrinkling. The typical size of iPDMS slab was $I \times w \times t=60 \times 10 \times 1$ $\mathrm{mm}^{3}$. The inserted red arrow indicated the direction of stretching; (b) Scanning electron microscopy images of wrinkles; (c) Atomic force microscopy image in height; (d) height profile corresponding to the white line in c; (e) iPDMS slab was stretched from $\mathrm{S}_{0}$ to S. For avoiding confusing with / above, letter " $\mathrm{S}$ " was chosen here. ( $\mathrm{f}-\mathrm{i})$ Factors that determined the wavelength of wrinkles. The typical experimental condition was applied except that one factor was varied: (f) varied $R_{\text {initiator }}$ whereas all other conditions were kept the same, that is, surface initiated polymerization (SIP) time was $3 \mathrm{~h}$ for oligo (ethylene glycol) methyl ether methacrylates (OEGMA) and $10 \mathrm{~min}$ for HEMA, respectively, $\varepsilon=2.0$; $(\mathrm{g})$ varied $\varepsilon$ whereas all other conditions were kept the same, that is, $\mathrm{R}_{\text {initiator }}=0.5$; (h) monomer OEGMA; and (i) monomer HEMA with varied SIP time whereas all other conditions were kept the same, that is, $\varepsilon=2.0, \mathrm{R}_{\text {initiator }}=0.5$. The black square stands for OEGMA and the red circle for HEMA. 
回
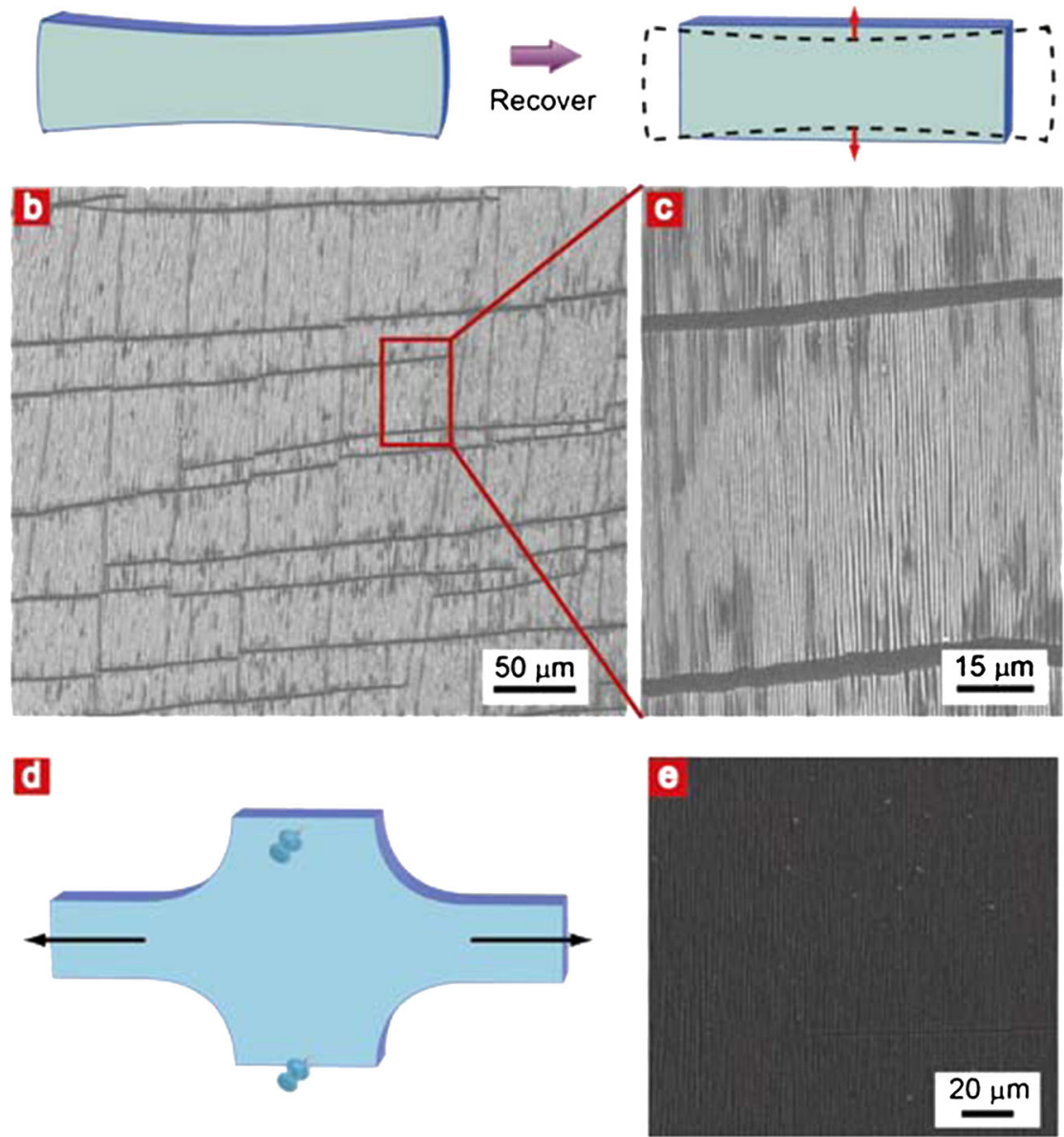

Figure 2. Proposed mechanism for crack formation and reduced number of cracks in the sample from subdued Poisson's effect (i.e., subdued lateral extension). (a) Stretching caused the decrease of width of integrated poly(dimethylsiloxane) (iPDMS) slab (i.e., lateral inward contraction) and lateral outward extension upon recovery; (b-c) scanning electron microscopy (SEM) images of cracks in crosslinked upper-skin under different magnification power. The crack lines were parallel to the stretching direction and perpendicular to the shrinkage direction of width; (d) an iPDMS slab was first stretched with settled lateral side for protecting it from Poisson's effect. After the generation of crosslinked upper-skin via surface initiated polymerization, the stretched iPDMS was allowed to recover; (e) SEM image proved that the number of cracks reduced significantly.

could switch between the continuous and discontinuous states via a simple stretching and release cycle, was proposed to interpret the appearance of cracks with crosslinker. In this model, the effect of transesterification between polymer chains was ignored, because the addition of methanol, which was used in our system, would suppress transesterification. ${ }^{[26]}$

The fact that this iPDMS/SIP system could survive $\varepsilon$ as high as 2.2 allowed us to directly monitor the evolution of wrinkle formation under optical microscope. We first chose a marker as the location identification as shown at the right upper corner of Fig. 3a. From Fig. 3a-d, as the value of $\varepsilon$ decreased from 1.8 to 1.1 , one observed the dynamics of wrinkle development. The onset of wrinkling was randomly distributed, indicating that iPDMS had a heterogeneous distribution of strain because wrinkles only started when the critical strain was reached. ${ }^{[5]}$ We highlighted three zones to follow as indicated with capital letters A-C. Each zone had parallel ridges and trenches that roughly perpendicular to the stretching direction (the red double-head arrow in Fig. 3a). As $\varepsilon$ decreased, the chosen zones spread along the direction of ridges but shrunk against the direction of stretching accompanied by the narrowing and prolonging of ridges and trenches with subtle adjustments in direction.
We attributed the fork formation to the randomness of the onset of wrinkling. Because the value of $\lambda$ is determined by the system (i.e., obey Eqn. (1)), the "Y" shaped forks must form if the distance between two onset points of wrinkling do not satisfy the $n \lambda$ rule. Thus, forks were usually produced only at the boundary of adjacent zones. It was further noticed that the sites of forks could be identified at very early stage of wrinkling: the red rings in Fig. 3b predicted the forks in Fig. 3d.

The elastomer nature of iPDMS further allowed us to repeat the stretching and recovery cycles, which is hard to achieve with fragile upper-skin and weak link in bilayer, so the following revealed wrinkling mechanisms have not been found to be reported before. After several circles of stretching and recovery (Fig. 3e), we found that the location of forks were unchanged, which was unexpected because the home-made device was unable to avoid unstable force distribution and other possible random noises. The ability of iPDMS/SIP system to remember the fork sites implied that some possible plastic deformation of upper-skin during the first recovery or the onset of wrinkling might be a direct consequence of heterogenity of mechanical properties of the iPDMS network, which was unlikely to change during the stretching and recovery cycles. The later reason was agreed 

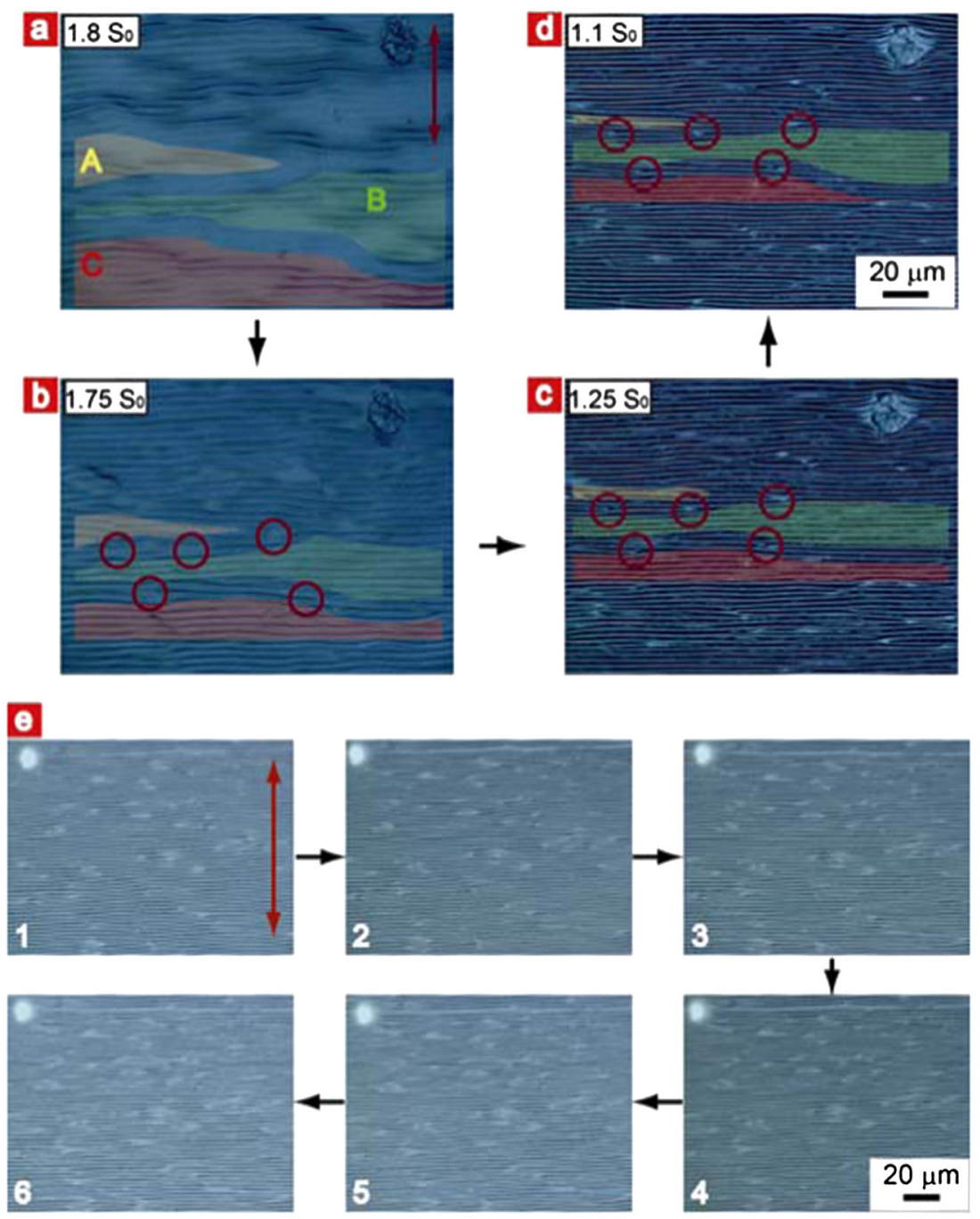

Figure 3. Snapshots of wrinkling dynamics under optical microscope and memory effect of integrated poly(dimethylsiloxane) (iPDMS) wrinkling. Stretching was gradually released from $2.0 \mathrm{~S}_{0}$ to (d) $1.8 \mathrm{~S}_{0}$, (e) $1.75 \mathrm{~S}_{0}$, (f) $1.25 \mathrm{~S}_{0}$, and (g) $1.1 \mathrm{~S}_{0}$. The locations of forks (marked by red circles) were determined at the early stage of wrinkling. (e)Twenty minutes of surface initiated polymerization of 2-hydroxyethyl methacrylate (HEMA) from $2.0 \mathrm{~S}_{0}$ iPDMS resulted in wrinkling as shown in the first picture. The same poly (HEMA)/iPDMS system was repeatedly stretched and released. The final states of wrinkling were recorded by optical microscope. A defect at the upper left corner was used as a landmark. Pattern and forks distributions were similar for all six times of stretching and release, indicating memory effect of iPDMS wrinkling.

with the phenomenon of randomly distributed onset of wrinkling that was discussed in an earlier paragraph. It will be interesting to see if such method could be developed to map the homogeneity of materials.

\section{CONCLUSIONS}

To summarize, this system was different from most previously reported systems in that (i) the reported iPDMS/SIP system could adopt $\varepsilon$ as high as 2.2 while still maintain a low level of crack defects. The uncrosslinked polymer skin was proved to be effective in reducing/eliminating crack defects; (ii) the upper-skin and foundation were connected by covalent bonds. This feature makes the skin/foundation bilayer more stable to avoid delamination ${ }^{[27]}$ and further allows us to dynamically and repeatedly stretching and releasing, which was key to reveal additional wrinkling mechanisms. We believe that this iPDMS/SIP system provides a novel and powerful system for wrinkle fabrications as well as important insights of the wrinkling mechanisms, which is the key to achieve wrinkles with better quality and finally realize its applications.

\section{Acknowledgements}

This work was supported by 100 Talents Programmer of CAS (08BM031001), the CAS/SAFEA International Partnership Program for Creative Research Teams, the NSFC grant (50773001), and Fok Ying Tung Education Foundation (114013) to H.M. The authors thank Mr. Yuanzi Wu, and Miss Yiman Jia for technical assistance and the electroscope support of Public Center for Characterization and Test, SINANO, CAS.

\section{REFERENCES}

[1] E. Cerda, L. Mahadevan, Phys. Rev. Lett. 2003, 90.

[2] J. Genzer, J. Groenewold, Soft Matter 2006, 2, 310.

[3] J. Yin, Z. X. Cao, C. R. Li, I. Sheinman, X. Chen, Proc. Natl. Acad. Sci. 2008, 105, 19132.

[4] A. Concha, J. W. Mclver, P. Mellado, D. Clarke, O. Tchernyshyov, R. L. Leheny, Phys. Rev. E Stat. Nonlin. Soft Matter Phys. 2007, 75, 016609. 
[5] N. Bowden, S. Brittain, A. G. Evans, J. W. Hutchinson, G. M. Whitesides, Nature 1998, 393, 146.

[6] K. Efimenko, M. Rackaitis, E. Manias, A. Vaziri, L. Mahadevan, J. Genzer, Nat. Mater. 2005, 4, 293.

[7] C. J. Yu, C. Masarapu, J. P. Rong, B. Q. Wei, H. Q. Jiang, Adv. Mater. 2009, 21, 4793.

[8] C. H. Lu, H. Mohwald, A. Fery, Soft Matter 2007, 3, 1530.

[9] F. Katzenberg, Macromol. Mater. Eng. 2001, 286, 26.

[10] J. Y. Chung, A. J. Nolte, C. M. Stafford, Adv. Mater. 2009, 21, 1358.

[11] P. F. Zhang, D. Y. Yang, Z. Li, H. W. Ma, Soft Matter 2010, 6, 4580.

[12] C. M. Stafford, C. Harrison, K. L. Beers, A. Karim, E. J. Amis, M. R. Vanlandingham, H. C. Kim, W. Volksen, R. D. Miller, E. E. Simonyi, Nat. Mater. 2004, 3, 545.

[13] H. Huang, J. Y. Chung, A. J. Nolte, C. M. Stafford, Chem. Mater. 2007, $19,6555$.

[14] J. Huang, M. Juszkiewicz, W. H. de Jeu, E. Cerda, T. Emrick, N. Menon, T. P. Russell, Science 2007, 317, 650.

[15] Y. G. Sun, W. M. Choi, H. Q. Jiang, Y. G. Y. Huang, J. A. Rogers, Nat. Nanotechnol. 2006, 1, 201.
[16] D. Y. Khang, H. Q. Jiang, Y. Huang, J. A. Rogers, Science 2006, 311, 208.

[17] E. P. Chan, A. J. Crosby, Adv. Mater. 2006, 18, 3238.

[18] W. H. Koo, S. M. Jeong, F. Araoka, K. Ishikawa, S. Nishimura, T. Toyooka, H. Takezoe, Nat. Photonics 2010, 4, 222.

[19] A. Chiche, C. M. Stafford, J. T. Cabral, Soft Matter 2008, 4, 2360.

[20] J. Y. Chung, J. P. Youngblood, C. M. Stafford, Soft Matter 2007, 3, 1163.

[21] T. Ohzono, H. Watanabe, R. Vendamme, C. Kamaga, T. Kunitake, T. Ishihara, M. Shimomura, Adv. Mater. 2007, 19, 3229.

[22] C. B. Lin, C. C. Lin, S. Lee, Y. T. Chou, J. Appl. Phys. 2008, 104.

[23] Y. Z. Wu, Y. Y. Huang, H. W. Ma, J. Am. Chem. Soc. 2007, 129, 7226.

[24] T. C. Qian, Y. F. Li, Y. Z. Wu, B. Zheng, H. W. Ma, Macromolecules 2008, 41, 6641 .

[25] Z. Li, D. Y. Yang, X. Liu, H. W. Ma, Macromol. Rapid Commun. 2009, 30, 1549.

[26] K. L. Robinson, M. A. Khan, M. V. D. Banez, X. S. Wang, S. P. Armes, Macromolecules 2001, 34, 3155.

[27] S. Edmondson, K. Frieda, J. E. Comrie, P. R. Onck, W. T. S. Huck, Adv. Mater. 2006, 18, 724. 